\title{
Ultra-fast conductive media for RNA electrophoretic mobility shift assays
}

\author{
Samantha Z Brown', Lebaron C Agostini', Henry L Thomsett' \& Jonathan R Brody*,1
}

\begin{abstract}
The use of RNA electrophoretic mobility shift assays (REMSAs) for analysis of RNA-protein interactions have been limited to lengthy assay time and qualitative assessment. To vastly improve assay efficiency, feasibility and quality of data procured from REMSAs, we combine here some of the best-known labeling and electrophoretic techniques. Nucleic acid fragments are end-labeled with fluorescent tags, as opposed to the radioactive or biotin tags. The fluorescent probes may be detected directly from the electrophoresis gel, eliminating the need for cumbersome membrane transfer and immunoblotting. Modifying the REMSA protocol to include low-molarity, lithium borate conductive media and near-infraredlabeled probes allows for a reduction assay time, quantitative comparison between experimental conditions and crisp band resolution (i.e., optimized results).
\end{abstract}

\section{METHOD SUMMARY}

We present an improvement in resolution, speed and ease of RNA electrophoretic mobility shift assays. First, sensitive and quantitative detection of gel shifts can be improved with near-infrared tagged RNA oligos, as opposed to more toxic and cumbersome labeling methods, such as radioisotopes or biotin tags. Second, for improvement of resolution and efficiency, traditional Tris-based conductive running media are replaced with low-molarity, lithium borate-conductive media. These improvements to the methodology significantly reduce assay time, as well as improve the quality and overall utility of this technique in the study of RNA-protein interactions in vitro.

\section{KEYWORDS}

conductive media $\cdot \mathrm{ELAVL1} \cdot \mathrm{EMSA} \cdot \mathrm{HuR}$ - IR-oligonucleotides $\cdot \mathrm{LB} \cdot \mathrm{RBP} \cdot \mathrm{REMSA}$

'Department of Surgery, The Jefferson Pancreas, Biliary \& Related Cancer Center, Thomas Jefferson University, Philadelphia, PA 19107, USA; *Author for correspondence: jonathan.brody@jefferson.edu

BioTechniques 68: 101-105 (February 2020) 10.2144/ btn-2019-0111
Gel electrophoresis mobility shift assay (EMSA) is a long-established biochemical technique for the qualitative assessment of nucleotide-protein complexes [1-4]. This method combines the principles of protein and oligonucleotide electrophoresis to determine biochemical relationships between these species. Ribonucleotide-based EMSAs (REMSAs) are a modified version of this technique to evaluate ribonucleotide-protein complexes. Most often, REMSAs are employed for the validation of RNA-binding proteins (RBPs) to their regulated transcripts.

Although the current methodology of this application is informative, its output is largely qualitative, with a variety of resolution and sensitivity issues. Earlier renditions of this method used radioisotopes such as ${ }^{32} \mathrm{P}$ for tagging oligonucleotides (oligos). Radioactive labeling is a labor-intensive process, resulting in issues with efficiency, cost and safety [2,5-7]. To address these challenges, one major improvement has replaced radioisotope tagging with biotin conjugation. This labeling technique relies on its affinity against avidinbased proteins for specific isolation of complexes [8-10].

Biotin end-labeled probes are easier and safer to use than radioisotope-based applications; however, this method still requires additional time-consuming steps after initial electrophoresis. Notably, after separating transcripts on a nondenaturing gel, contents must be cross-linked and transferred to a matrix membrane. Anti-streptavidin-HRP conjugate and substrate are then needed to develop the blots and visualize gel shifts. These additional steps not only add to the overall process time (i.e., 4-6 h), but also add extra materials, cost and optimization time.

To further address the limitations of prior assays, we end-labeled RNA transcripts with near-infrared (NIR) fluorescent dyes $[4,11]$. RNA oligos were ordered and synthesized from integrated DNA technologies (IDT, IA, USA) (Figure 1A). The specific NIR dyes and sequences of the transcripts that were used in the following experiments are described in Table 1. After electrophoresis, gels were immediately imaged on an NIR scanner (LI-COR Odyssey scanner, NE, USA). The sensitivity of these conjugated dyes allows for quantitative analysis of bound and unbound probes (Figure 1B). Although not demonstrated here, the minimum detectable concentrations of probes may be optimized to save materials and to visualize proteinoligonucleotide association. The cost of synthesizing each NIR-conjugated probe is roughly US\$100 more than a biotinlabeled probe; however, considering that the fluorescent signal can be quantitated directly, gel transfer to a membrane is not necessary to visualize samples, and, therefore, provides a more time-efficient and economical option.

To demonstrate the function of these probes within biologic contexts, we used a defined RBP-ribonucleic acid relationship between the RBP, HuR/ ELAVL1 and one of its mRNA targets, COX-2 [12,13]. NIR probe concentrations were optimized through titration experiments (Figure 1B). For these experiments, total protein was either extracted from crude cell lysate under nondenaturing conditions, or purchased as recombinant protein (Origene, MD, USA). To determine optimal protein concentration (i.e., whole-cell extracts or recombinant proteins), a fixed probe concentration was incubated with titrating amounts of lysate to visualize a gel shift (i.e., clear interpretable shifts in the bound probe as compared with the unbound probe) (Figure 1C\&D). Nontargeted recombinant tRNA (Thermo Fisher Scientific, NE, USA) was included in to reduce nonspecific 
(A)

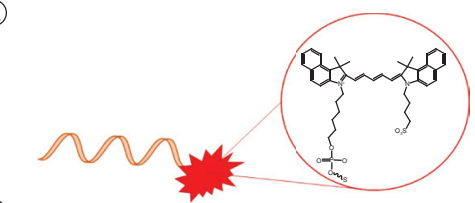

(C)
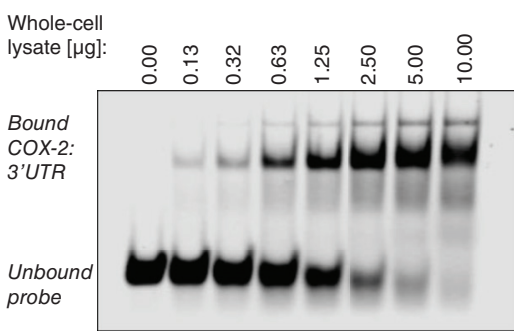

Bound

WEE1:

EXON2

Unbound

probe

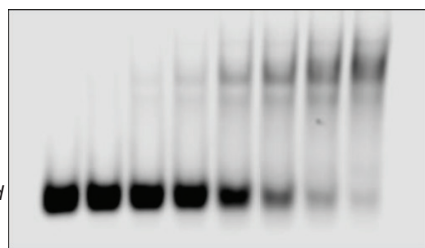

(B)

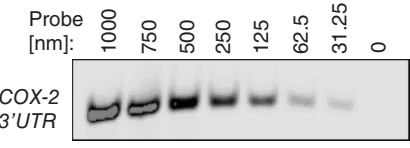

(D)

Recombinant protein $[\mu \mathrm{g}]$
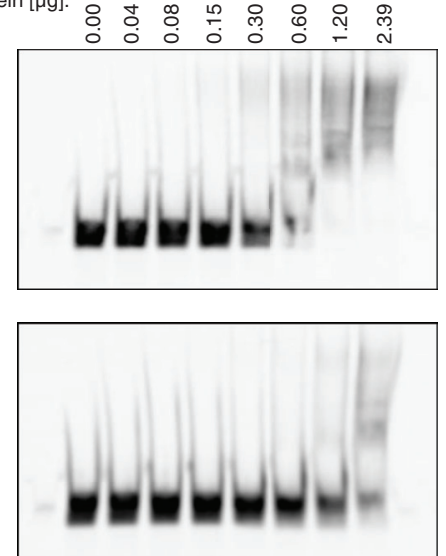

Figure 1. Near-infrared-labeled oligonucleotides provide sensitive and quantitative detection of protein-RNA complexes. (A) RNA oligonucleotides synthesized with near-infrared (NIR) labels by Integrated DNA Technologies (IA, USA). (B) (1:1) Titration of [1 $\mu \mathrm{M}]$ IR-labeled probe in $1 \times$ LB running buffer. (C \& D) A fixed concentration of NIR-labeled probe [62.5 nM] run with titrated nondenaturing, crude-cell lysate versus recombinant HuR (Origene, MD, USA). Reactions run at $250 \mathrm{~V}$ for $10 \mathrm{~min}$ at room temperature. Gels were imaged directly on an infrared scanner (Licor, NE, USA) at a wavelength corresponding to the tagged NIR dye $(690 \mathrm{~nm})$. All gels comprised $1 \times$ LB (MD, USA), $5 \%$ acrylamide/bis, $10 \%$ APS, and TEMED (BioRad, CA, USA). Data in (C \& D) are representative of at least two separate experiments.

APS: Ammonium persulfate; LB: Lithium boric acid; NIR: Near-infrared; TEMED: Tetramethylethylenediamine.

binding between probes and HuR (Figure 1C \& D). Complexes were incubated at room temperature for $30 \mathrm{~min}$ in the dark before being loaded in a $5 \%$ weight by volume $(\mathrm{w} / \mathrm{v})$ acrylamide gel. Table 2 outlines specific reaction components and concentrations are outlined in. Table 3 describes assay buffers in depth.

The second limitation of former REMSA methods is the use of Tris-based (Tris-HCL/ Boric Acid/EDTA, TBE) conductive media, most likely adapted from early electropho- retic methods [10]. Because of its poor conductivity and high heat of reaction owing to Ohm's law, TBE causes poor oligonucleotide resolution $[9,10]$. For this reason, TBE gels must be run for long stretches of time (>90 min) at low voltages to maintain gel-complex integrity. To resolve resolution issues and improve the reaction time, we exchanged TBE (Fisher Scientific, NH, USA) for a more effective low-molarity conductive media, lithium boric acid (LB) (Faster Better Media, LLC, MD, USA) [8]. It has long been demonstrated that agarosebased electrophoresis LB media is able to rapidly separate DNA and RNA species at high voltages, without compromising resolution or sensitivity [8-10]. Moreover, the cost of 1 liter of $10 \times$ TBE is the same as commercial liter bottles of $10 \times$ LB buffer at roughly US\$50 unit purchase. To evaluate the improved buffering capacity of the LB method, $5 \% \mathrm{w} / \mathrm{v}$ gels were cast and ran with $1 \times$ TBE or LB medium, as indicated (Table 3). The temperature, current and integrity of the gel were evaluated at fixed run times and at high voltage (300 V) (Figure 2). Under each run time, LB was able to separate oligos with enhanced resolution, demonstrating a more pronounced gel shift, as indicated by clear separation of 'probe only' and 'probe plus HuR protein' lanes (Figure 2). As expected, the final temperatures of the TBE gel were elevated over the LB-based method, despite similar volumes of media used and equal thickness of the gels prepared $[9,10]$. When we separated the glass gel sandwich, we noted that the physical integrity of the TBE gel was significantly impaired compared with the solid matrix of the LB gel. In addition, we noted that the TBE gels showed an increased temperature of $5^{\circ} \mathrm{C}$ over the running of the assay, whereas LB gels increased roughly $3^{\circ} \mathrm{C}$ or lower. In terms of conductance, LB gels ran at a constant 30 milliamps, whereas the TBE gels rose from 40 to 80 milliamps. This expected relationship between conductance and temperature was previously described in agarose gel electrophoresis systems $[10,12]$. Based on these results, we demonstrate that higher voltages over a shorter period of time are possible without deleterious temperature increases that may impact gel integrity and assay resolution (Figure 2). Here, we refer to this improved protocol as lithium-based, near-infrared REMSA (LI-REMSA).

\section{Table 1. Near-infrared labeled RNA oligonucleotides.}

\begin{tabular}{|l|l|l|}
\hline Name & Sequence & \multicolumn{2}{|c|}{ Use } \\
\hline $\begin{array}{l}\text { COX2-3'UTR } \\
\text { (49 bps) }\end{array}$ & $5^{\prime}$-/5IRD700/UCUAUUAAUUUAAUUAUUUAAUAAUAUUUAUAUUAAACUCCUUAU-3' & Positive control \\
\hline $\begin{array}{l}\text { WEE1-EXON2 } \\
\text { (49 bps) }\end{array}$ & $5^{\prime} / 5$ IRD700/GAAACAGACCUGCUAAGGUGUGUGAAGAGGCUGGAUGGAUGCAGAACG-3' & Negative control \\
\hline WEE1-10T (49 bps) & 5'/5IRD700/UGUGUGUCCAUCUUAUAUUUCUUUUUUUUUUAAUUGUGAAUUAGACUU-3' & Experimental \\
\hline
\end{tabular}

bp: Base pair; NIR: Near-infrared; 3'UTR: 3'-Untranslated region. 


\begin{tabular}{|c|c|}
\hline Nondenaturing lysis buffer & Final concentration \\
\hline Tris- $\mathrm{HCl}(\mathrm{pH} 7.5)$ & $75 \mathrm{mM}$ \\
\hline Sodium chloride $(\mathrm{NaCl})$ & $150 \mathrm{mM}$ \\
\hline Magnesium chloride $\left(\mathrm{MgCl}_{2}\right)$ & $1 \mathrm{mM}$ \\
\hline Nonidet P-40 (or IGEPAL-CA630) & $1 \%(\mathrm{v} / \mathrm{v})$ \\
\hline Phenylmethane sulfonyl fluoride (PMSF) & $1 \mathrm{mM}$ \\
\hline Protease inhibitor cocktail (PI) & $1 \mathrm{mM}$ \\
\hline Sodium orthovanadate $\left(\mathrm{Na}_{3} \mathrm{VO}_{4}\right)$ & $1 \mathrm{mM}$ \\
\hline $5 \times$ binding buffer & Final concentration \\
\hline HEPES (pH 7.4) & $75 \mathrm{mM}$ \\
\hline Potassium chloride $(\mathrm{KCl})$ & $50 \mathrm{mM}$ \\
\hline Glycerol & $50 \%(v / v)$ \\
\hline Dithiothreitol (DTT) & $1 \mathrm{mM}$ \\
\hline Magnesium chloride $\left(\mathrm{MgCl}_{2}\right)$ & $25 \mathrm{mM}$ \\
\hline Binding reaction components & Final concentration \\
\hline tRNA & $5 \mu \mathrm{g}$ \\
\hline Fluorophore-tagged RNA & $\neq$ \\
\hline Protein lysate or recombinant protein & $\ddagger$ \\
\hline $5 \times$ binding buffer & $1 \times$ \\
\hline $5 \times$ loading dye & $1 \times$ \\
\hline
\end{tabular}

Figure 2. Improved resolution and assay time with lithium boric acid conductive media versus Tris-boric acid-disodium EDTA method.

(A) $1 \times$ TBE gel and (B) $1 \times$ LB gel ran fast (300 V) for $7 \mathrm{~min}$, as indicated. (C \& D) Gels may be run longer without compromising resolution, in which the same gels were returned to the reservoirs, and run for an additional $5 \mathrm{~min}$ (total assay time: $12 \mathrm{~min}$ ). After run time, gels were removed from the reservoirs and were imaged using infrared scanner (LICOR, NE, USA) at a wavelength corresponding to the tagged NIR dye $(690 \mathrm{~nm})$. Data shown in the figure are representative of at least three separate experiments. Gel temperatures were monitored by placing standard thermometer directly in reservoir before and after completion of the run. LB: Lithium boric acid; TBE: Tris-boric aciddisodium EDTA; $T_{0}$ : Initial temperature; $\mathrm{T}_{\mathrm{F}}$ : Final temperature.

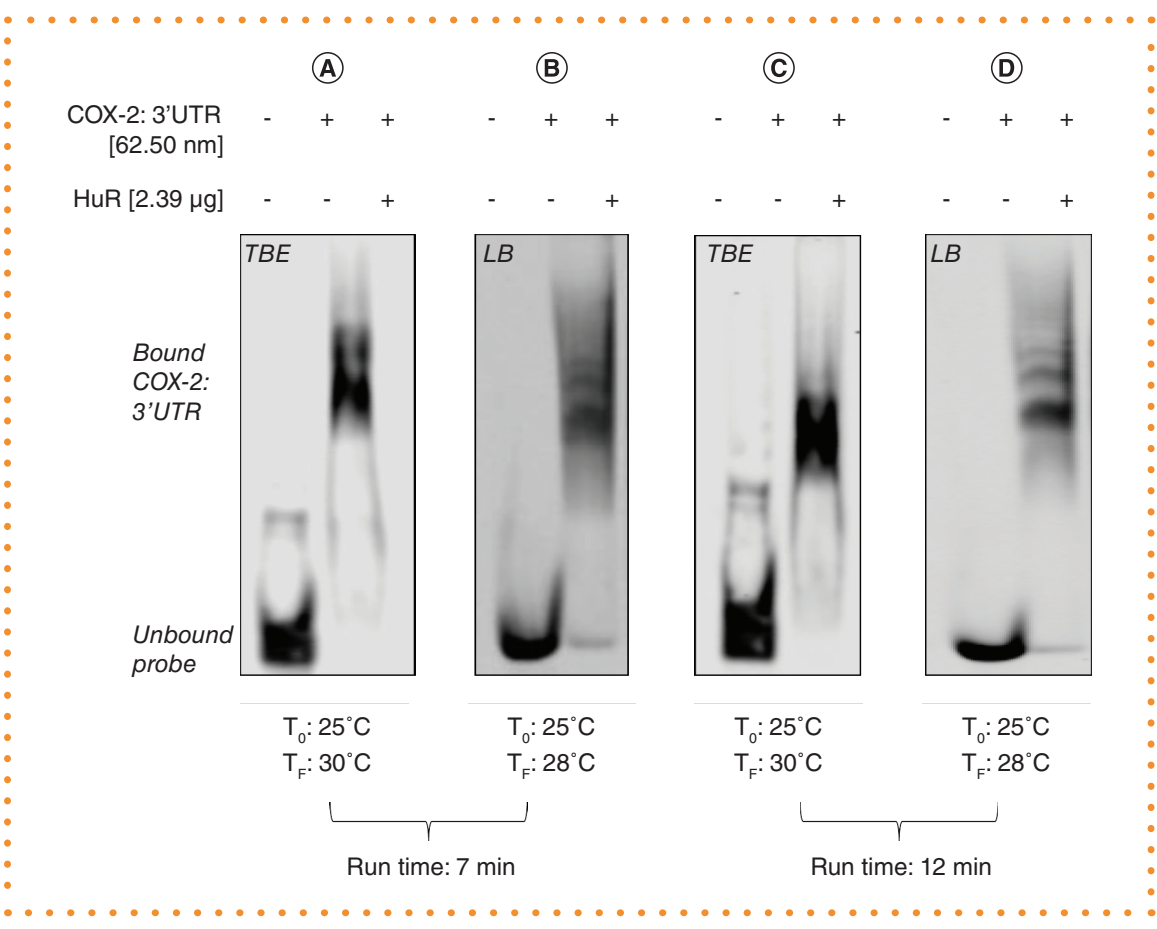


Table 3. Tris-boric acid-disodium EDTA versus lithium boric acid method components.

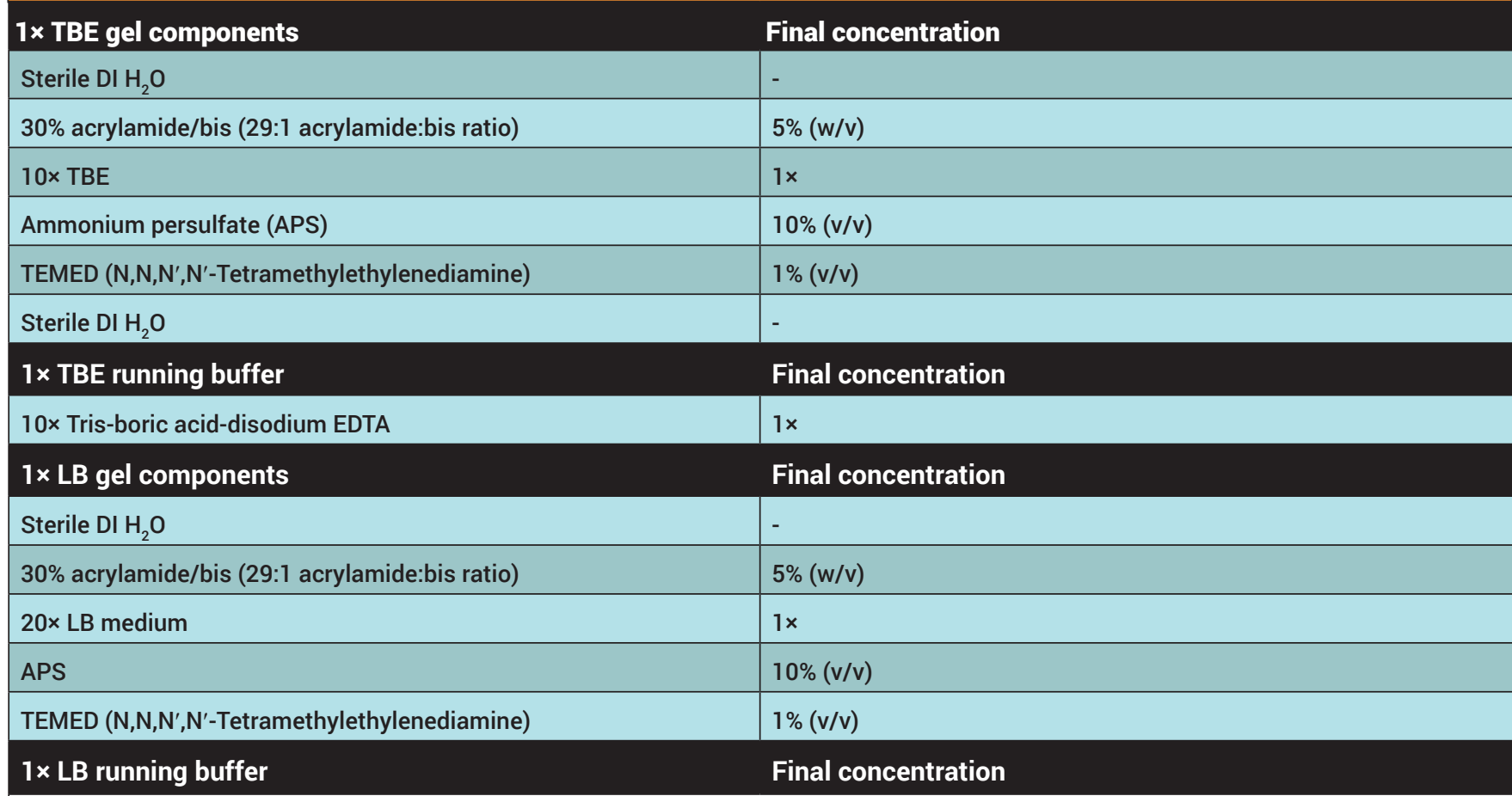

APS: Ammonium persulfate; LB: Lithium boric acid; TBE: Tris-boric acid-disodium EDTA; v/v: Volume by volume; w/v: Weight by volume

REMSAs are classically used for validation of RBP target sequences within an mRNA transcript of interest in vitro. Here, we validated a previously predicted binding site of a HuR-regulated transcript, WEE1 (the G2/M mitotic checkpoint inhibitor) mRNA, using LI-REMSA (Figure 3) [14]. Each probe was incubated with either recombinant HuR or FOXP3, a negative control transcription factor (i.e., that should not bind to WEE1
mRNA). A sequence within the second exon of the WEE1 mRNA was used as a negative control. The WEE1 Exon2 mRNA probe, which lacks adenylate-uridylaterich elements required for HuR recognition, demonstrated unresolved resolution with a large percentage of unbound free probe, indicating weak to no binding in these assay conditions (Figure 3). Notably, the 3' UTR WEE1 probe showed significant protein binding, with no evidence of unbound, 'free' target at concentrations used in this assay (Figure 3).

This work optimizes an existing method of RBP-ribonucleotide assessment by improving band resolution and decreasing total process time over traditional and more recent REMSA methods (i.e., total assay time $<1 \mathrm{~h}$ ). Moreover, the examples here can be expanded or modified to address experi-
Figure 3. LI-RNA electrophoretic mobility shift assays used for detection and validation of new RNA-binding protein target sequences. NIR-labeled probes were incubated in binding buffer with recombinant FOXP3 (negative control protein), HuR or none as indicated above (Origene, MD, USA). A total of $5 \mu \mathrm{g}$ of tRNA (Thermo Fisher Scientific, MA, USA) were used for each reaction to control for nonspecific binding. The $1 \times$ LB gels were run for $10 \mathrm{~min}$ at $250 \mathrm{~V}$. Data are representative of at least two separate experiments.

LB: Lithium boric acid; LI-REMSA: Lithiumbased, near infrared RNA electrophoretic mobility shift assay; NIR: Near infrared; 3'UTR: 3'-Untranslated region. 
mental needs based on the interactions being investigated. Others have shown the feasibility of additional end-labeled probes (i.e., alternative dyes) for assessment of competition or kinetics [5,15]. Furthermore, when using recombinant proteins for in vitro determination of binding to RNA sequences, electrophoresed gels do not need to be transferred for visualization of bound complexes in our assay protocol. The modifications presented here optimize the REMSA method, particularly in process time and ease of use.

\section{AUTHOR CONTRIBUTIONS}

SZ Brown and HL Thomsett conceived the presented data. SZ Brown and LC Agostini carried out the experiments. SZ Brown wrote the final manuscript. JR Brody provided funding, critical feedback and helped shape the research. All authors discussed the results and contributed edits to the final version.

\section{FINANCIAL \& COMPETING INTERESTS DISCLOSURE}

This work was supported by National Institutes of Health grant number 1R01CA212600-01, and the Sarah Parvin Foundation. JR Brody is the co-owner of Faster Better Media LLC and as such has received revenue on sales of products described in this article. This creates a significant financial conflict of interest, which is being managed by The Johns Hopkins University and Jefferson University in accordance with its conflict of interest policies. The authors have no other relevant affiliations or financial involvement with any organization or entity with a financial interest in or financial conflict with the subject matter or materials discussed in the manuscript apart from those disclosed.

No writing assistance was utilized in the production of this manuscript.

\section{OPEN ACCESS}

This work is licensed under the AttributionNonCommercial-NoDerivatives 4.0 Unported License. To view a copy of this license, visit http://creativecommons.org/licenses/ by-nc-nd/4.0/

\section{REFERENCES}

Papers of special note have been highlighted as: • of interest; • • of considerable interest

1. Garner MM, Revzin A. The use of gel electrophoresis to detect and study nucleic acid-protein interactions. Trends Biochem. Sci. 11(10), 395-396 (1986).

- Founding paper on gel electrophoresis principles for the separation of nucleic acids and proteins together in solution.

2. Fried MG. Measurement of protein-DNA interaction parameters by electrophoresis mobility shift assay. Electrophoresis 10(5-6), 366-376 (1989).

- Founding paper on gel electrophoresis principles for the separation of nucleic acids and proteins together in solution.

3. Rio DC. Electrophoretic mobility shift assays for RNAprotein complexes. Cold Spring Harb. Protoc. 2014(4), 435-440 (2014).

- Founding paper on gel electrophoresis principles for the separation of nucleic acids and proteins together in solution.

4. Hsieh YW, Alqadah A, Chuang CF. An optimized protocol for electrophoretic mobility shift assay using infrared fluorescent dye-labeled oligonucleotides. J. Vis. Exp. doi:10.3791/54863(117) (2016) (Epub ahead of print).

.. Important principle paper for explaining the concept of fluorescence-based shift assays.

5. Hellman LM, Fried MG. Electrophoretic mobility shift assay (EMSA) for detecting protein-nucleic acid interactions. Nat. Protoc. 2(8), 1849-1861 (2007).

Overview of electrophoretic mobility shift assay principles with outline of important technical steps.

6. Fillebeen C, Wilkinson N, Pantopoulos K. Electrophoretic mobility shift assay (EMSA) for the study of RNA- protein interactions: the IRE/IRP example. J. Vis. Exp. do $\mathrm{i}: 10.3791 / 52230$ (94) (2014) (Epub ahead of print).

- Overview of RNA electrophoretic mobility shift assays principles with outline of important technical steps.

7. Poulin-Laprade D, Burrus V. Electrophoretic mobility shift assay using radiolabeled DNA probes. Methods Mol. Biol. 1334, 1-15 (2015).

- Complete overview of the radiolabeled EMSA method technique and principles.

8. Brody JR, Calhoun ES, Gallmeier E, Creavalle TD, Kern SE. Ultra-fast high-resolution agarose electrophoresis of DNA and RNA using low-molarity conductive media. BioTechniques 37(4), 598, 600, 602 (2004).

.. Principle paper for discovering and highlighting the advantages of low-molarity (LB) media over the traditional tris-based method.

9. Brody JR, Kern SE. Sodium boric acid: a Tris-free, cooler conductive medium for DNA electrophoresis. BioTechniques 36(2), 214-216 (2004)

- Principle paper for discovering and highlighting the advantages of a Tris-free (sodium boric acid) media over the traditional tris-based method.

10. Brody JR, Kern SE. History and principles of conductive media for standard DNA electrophoresis. Anal. Biochem. 333(1), 1-13 (2004).

-. Principle paper surveying the history and guiding principles behind the advantages and limitations of different conductive media.

11. Ruscher K, Reuter M, Kupper D, Trendelenburg G, Dirnag $\mathrm{U}$, Meisel A. A fluorescence based non-radioactive electrophoretic mobility shift assay. J. Biotechnol. 78(2), 163-170 (2000).

-. Important founding paper for explaining the concept of fluorescence-based shift assays.

12. Sengupta $S$, Jang BC, Wu MT, Paik JH, Furneaux $H$, Hla T. The RNA-binding protein HuR regulates the expression of cyclooxygenase-2. J. Biol. Chem. 278(27), 25227-25233 (2003).

- Citation for the validated, positive control as an HuR target in these experiments.

13. Peng SS, Chen $C Y, X u N$, Shyu AB. RNA stabilization by the AU-rich element binding protein, HuR, an ELAV protein. EMBO J. 17(12), 3461-3470 (1998).

- Citation for ELAVL1/HuR as the primary protein exemplified in this technique.

14. Lal S, Zarei M, Chand SN et al. WEE1 inhibition in pancreatic cancer cells is dependent on DNA repair status in a context dependent manner. Sci. Rep. 6 , 33323 (2016).

Citation for the negative control, and the experimental target, WEE1, as an HuR in these experiments and highlighting the biologic importance of HuR.

15. Gerstle JT, Fried MG. Measurement of binding kinetics using the gel electrophoresis mobility shift assay. Electrophoresis 14(8), 725-731 (1993).

- Understanding applications of gel electrophoresis for assessing oligonucleotide binding kinetics. 